Cahiers $d u$ MONDE RUSSE

\section{Cahiers du monde russe}

Russie - Empire russe - Union soviétique et États indépendants

40/3 | 1999

Varia

\title{
Premiers pas des archéologues russes et français dans le Turkestan russe (1870-1890)
}

Méthodes de recherche et destin des collections

\section{Svetlana Gorshenina}

\section{(2) OpenEdition}

Journals

Édition électronique

URL : https://journals.openedition.org/monderusse/17

DOI : $10.4000 /$ monderusse. 17

ISSN : $1777-5388$

Éditeur

Éditions de l'EHESS

Édition imprimée

Date de publication : 1 avril 1999

Pagination : 365-384

ISBN : 2-7132-1321-5

ISSN : $1252-6576$

Référence électronique

Svetlana Gorshenina, "Premiers pas des archéologues russes et français dans le Turkestan russe (1870-1890) », Cahiers du monde russe [En ligne], 40/3 | 1999, mis en ligne le 15 janvier 2007, consulté le 04 septembre 2022. URL : http://journals.openedition.org/monderusse/17 ; DOI : https://doi.org/ $10.4000 /$ monderusse. 17 


\title{
PREMIERS PAS DES ARCHÉOLOGUES RUSSES ET FRANÇAIS DANS LE TURKESTAN RUSSE (1870-1890)
}

\author{
Méthodes de recherche et destin des collections
}

\section{Les premières tentatives}

SAMARCANDE A TOUJOURS OCCupé unE POSITION ClÉ dans la longue succession des civilisations d'Asie Centrale, ce qui explique l'intérêt constant que lui portent les chercheurs. L'un des moments les plus attachants de son histoire, aussi bien pour les Russes que pour les Occidentaux, est la période liée au nom d'Alexandre le Grand. Les sources écrites, qui décrivent avec une relative précision le séjour à Marakanda de la garnison grecque, installée dans un endroit fortifié à l'intérieur de la ville (l' «acra » d'Arien ou l'arx de Quinte-Curce) $)^{1}$ disent que c'est là que la garnison grecque subit le siège de Spitamène et que se déroula un banquet au cours duquel fut tué Cléitos. La recherche des traces réelles de ces événements a été le point de départ de la première étape de l'exploration archéologique d'Afrasiab.

Les particularités de la situation géopolitique qui s'était constituée (intégration d'une partie de l'Asie Centrale dans l'Empire russe, établissement du protectorat russe sur le reste des territoires) ont conduit pratiquement à un monopole russe sur l'exploration archéologique de cette région. Pour cette raison les notes des chercheurs occidentaux (dont la durée de séjour et la possibilité même de séjourner au Turkestan dépendaient directement de la bonne volonté de l'administration russe) étaient souvent fragmentaires; elles comportaient de nombreuses imprécisions concernant la chronologie, la toponymie et l'onomastique et se rattachaient plutôt au genre de la description littéraire du « voyage en Orient ». Mais les publications

1. Arrien (en grec Arrhianos, latin Flavius Arianus), IV, 3, 6-7, Quinte-Curce, VII, 6, 10. 
russes ne dépassaient guère en qualité celles des archéologues occidentaux : la Russie manquait de véritables spécialistes dans le domaine de l'archéologie centrasiatique et accordait une priorité incontestable à l'étude des sites en Russie. En revanche, elles furent nettement supérieures en nombre en particulier à partir de 1868, date du rattachement du district de Samarcande au gouvernorat du Turkestan. D'autre part, les premières descriptions un tant soit peu détaillées faites par les orientalistes, géologues, naturalistes et explorateurs russes, bien que contenant des descriptions parfois assez précises des monuments de Samarcande, y compris d'Afrasiab, ne mettent pas particulièrement en relief les problèmes archéologiques ${ }^{2}$.

La construction à travers Afrasiab de la route Samarcande-Tachkent, entraînant de nombreuses découvertes, ainsi que la situation du marché des antiquités du Turkestan, de plus en plus inondé d'objets antiques de toutes sortes, ont conduit à mettre en œuvre des fouilles officiellement sanctionnées par le gouvernement russe, qui correspondent à la première étape de l'étude d'Afrasiab. En 1873, le chef de la région (okrug) du Zerafšan, le général-major A. K. Abramov, envoya en mission à Afrasiab le chef du district (otdel) de Samarcande, le major Borzenkov pour y mener des fouilles archéologiques. En 1883, sur l'ordre du général-gouverneur, M. G. Černjaev, des fouilles furent entreprises par un fonctionnaire, préposé aux missions spéciales, V. V. Krestovskij (connu par ailleurs comme écrivain). La troisième campagne de fouilles est liée au nom de N. I. Veselovskij, envoyé au Turkestan par la Commission archéologique de Saint-Pétersbourg en 1884 (il fit une autre campagne, moins importante, en 1895).

À ces études archéologiques de longue durée menées par la Russie, peuvent seules se comparer, par leur caractère et leur durée et pour la même période, les recherches du Français Jean Chaffanjon (1894-1895). Les autres chercheurs occidentaux, n'ayant pas eu la possibilité de mener des travaux de longue durée sur place, se sont limités à de brèves descriptions des monuments, rédigées au fil du voyage, ou bien, comme ce fut le cas pour Edouard Blanc (1893) et Charles-Eugène Ujfalvy (1879-1880), à des fouilles superficielles d'un jour, menées un peu au hasard ${ }^{3}$.

Il est important de noter que le tiers des chercheurs occidentaux qui ont exploré le Turkestan à cette époque étaient des Français. L'Alliance franco-russe était alors à son apogée, ce qui eut pour conséquence l'hospitalité inhabituelle des autorités russes, peu enclines d'ordinaire à autoriser aux chercheurs étrangers l'accès du Turkestan. Chaffanjon, lui, se vit accorder la possibilité de mener des fouilles, il

2. La mission des ingénieurs des mines, K. F. Butenev et F. Bogoslovskij avec la participation du naturaliste A. Leman, de l'orientaliste N. V. Hanykov et du topographe Jakovlev (1841), les expéditions de A. P. Fedčenko (1869-1870), l'expédition de V. V. Radlov (1869), l'expédition de I. V. Mušketov (années 70), etc.

3. Ch.-E. De Ujfalvy de Mezö-Kövesd, Le Syr-Daria. Le Zérafchane. Le pays des sept-rivières et la Sibérie occidentale, Paris, ed. Ernest Leroux, 1887, p. 81 ; E. Blanc, « Notes de voyage en Asie centrale. Samarkande », Revue des Deux Mondes, 115, 1893, p. 827. 
disposa, sur l'ordre du général A. N. Kuropatkin, d'un wagon spécialement équipé, il eut le droit de circuler librement tout au long de la voie ferrée transcaspienne et fut muni de cartes de la région, élaborées par les topographes de l'état-major général. Il obtint également l'autorisation de sortir ses collections du pays ${ }^{4}$.

Si les bases de départ différaient, les méthodes de recherche archéologiques comportaient beaucoup de pratiques communes, conditionnées par la problématique dominante du moment et le manque de perfectionnement des principes mêmes qui régissaient les recherches archéologiques.

Le souci principal de cette époque n'était pas la mise en lumière de la structure urbanistique de la ville ancienne et des étapes de sa formation, mais la découverte d'œuvres d'art sensationnelles, confirmant l'existence dans la région de civilisations anciennes. C'est pourquoi l'attention se concentrait surtout sur les endroits où la probabilité de découvertes intéressantes était la plus forte. On accordait la priorité à la partie centrale, la plus élevée, de la ville ancienne. V. A. Šiškin, s'appuyant sur sa longue expérience de travail à Afrasiab et sur les descriptions de M. Rostislavov 5 , écrivait que : « apparemment Borzenkov avait mené des fouilles à l'extrémité nord de la ville ancienne, peut-être dans la zone de la citadelle, là où les ondulations du sol sont les plus importantes ${ }^{6}$. Les travaux de Chaffanjon se concentrèrent essentiellement sur la citadelle. Ceux de V. V. Krestovskij s'étendirent à une butte à proximité de la route Samacande-Tachkent, alors que N. I. Veselovskij avait reçu de la Commission archéologique de Saint-Pétesrbourg la consigne de « se limiter à la fouille d'une seule partie de la ville ancienne, par exemple la citadelle, le palais ou la portion du site qui, après plus ample connaissance du milieu, semblerait présenter un intérêt particulier $»^{7}$. C'était également l'approche des

4. Chaffanjon lui-même indique que les officiers généraux russes et les services consulaires facilitèrent considérablement les recherches scientifiques de la mission française : le général N. A. Kaštalinskij, collectionneur passionné d'antiquités, montra aux Français les ruines de la ville antique de Merv; le capitaine Korol'kov qui commandait l'artillerie de Preževalsk leur révéla les résultats de près de trente ans d'observation de la dynamique du flux et du reflux des eaux du lac Issyk-Kul ; le général-gouverneur de la ville de Vernyj (l'actuelle Almaty), Ivanov, mit à la disposition de la mission les chiffres officiels montrant la progression de la colonisation de la région, ainsi qu'une série d'observations météorologiques ; en compagnie de N. A. Pantusov et de l'ingénieur Gourdet, Chaffanjon étudia le cimetière nestorien du faubourg de Vernyj ; les consuls et les agents commerciaux russes furent les premières personnes avec lesquelles le chercheur français entra en contact pendant son voyage à travers la Mongolie et la Chine. Cf. AN (Archives Nationales de France), fonds F 17/2946/2 ; J. Chaffanjon, « Rapport sur une mission scientifique dans l'Asie centrale et la Sibérie », in Nouvelles archives des missions scientifiques et littéraires, Paris, Impr. nationale, 1899, 9, pp. 55-56, 61, 63, 64, 69, 72, 77, 82.

5. M. Rostislavov, « Ob arheologičeskih izyskanijah v Zerafšanskom okruge » (Au sujet de recherches archéologiques dans la région du Zerafšan), Turkestanskie vedomosti, 27, 1875, réimpression : Protokoly Turkestanskogo kružka ljubitelej arheologii [Tachkent], 3, 18971898,pp. 144, 149.

6. V. A. Šiškin, «K istorii arheologičeskogo izučenija Samarkanda i ego okrestnostej » (Contribution à l'histoire de l'étude archéologique de Samarcande et de ses environs), in Afrasiab, Tachkent, Fan, 1969, 1, p. 11.

7. Citation d'après V. A. Šiškin, art. cit., p. 25. 
archéologues français qui travaillaient dans d'autres régions d'Asie (Henri de Morgan à Suse et Alfred Foucher en Afghanistan $)^{8}$. Cette approche reposait sur la conviction que c'était justement dans la citadelle que l'on pouvait trouver les objets les plus intéressants, susceptibles de fournir une clé pour la compréhension des cités anciennes ; on n'avait nullement conscience à l'époque que la citadelle représentait le secteur de fouille le plus complexe et le moins prometteur de succès rapides ${ }^{9}$.

Les méthodes de fouille n'étaient pas très variées ni très élaborées. On entreprenait les travaux « en creusant deux corridors en croix », sans la moindre notation des plans et des coupes et sans tenir de journal des observations (Borzenkov) ${ }^{10}$; ou bien on creusait une tranchée assez large (plus de trois mètres) qui était elle-même coupée de tranchées transversales plus étroites (Krestovskij). Il faut dire que V. V. Krestovskij, qui (à la différence de tous les autres) avait soigneusement noté toutes les étapes du travail ${ }^{11}$, en vint par la suite à la conclusion qu'il était indispensable de mener les fouilles du chantier couches par couches et il en fixa le nombre à dix.

Pour sa part, N. I. Veselovskij avait reçu, dès le début des travaux, des indications précises de la Commission archéologique sur la nécessité de procéder à la fouille du site par couches. Cependant sa méthode représente un retour en arrière par rapport à celle de V. V. Krestovskij. Il refusa de procéder à la fouille par couches et en un seul point du site, décidant

«de parcourir tout Afrasiab, pour choisir ensuite les endroits les plus intéressants [...]. Ayant commencé les travaux à l'ouest de la citadelle, il traça un grand demi-cercle en direction du tombeau de Danijar, creusant de nombreuses tranchées et coupes, englobant dans ses travaux la citadelle et pratiquant même une fouille superficielle hors de la ville ancienne sur Bujrobazar (le marché des nattes) situé près de l'angle sud-ouest de la ville ancienne. $»^{12}$

En quatre mois il pratiqua 109 fouilles.

Mais la figure la plus détestable de la première période d'exploration d'Afrasiab, si l'on en croit la tradition archéologique soviétique ${ }^{13}$, est celle du Français

8. F. Olivier-Utard, Politique et archéologie. Histoire de la délégation archéologique française en Afghanistan (1922-1982), Paris, Ministère des Affaires Étrangères, Ed. Recherches sur les civilisations, 1997, p. 72. Voir aussi le compte rendu critique de Frantz Grenet dans le Bulletin of the School of Oriental and African Studies, 62, 1, 1999, pp. 159-160.

9. Pour une critique de cette méthode, cf. M. Wheeler, Archaeology from the earth, Oxford, Clarendon Press, 1955, XI, 221 p.

10. M. Rostislavov, art. cit.

11. V. Krestovskij, « Samarkandskie raskopki 1883 g. » (Les fouilles de Samarcande en 1883), Sankt-Peterburgskie vedomosti, 32, 34, 35, 36, 37, 1984. Cf. également au sujet de ses fouilles Turkestanskie vedomosti, 42, 1883 ; 1, 1884 ; Syn Otečestva, 288, 1883 ; Novoe vremja, 2741, 1883.

12. V. A. Šiškin, art. cit., p. 27.

13. M. E. Masson, « Kratkij očerk istorii izučenija Srednej Azii v arheologičeskom otnošenii » (Court traité d'histoire de l'étude de l'Asie Centrale d'un point de vue archéologique), Trudy Sredneaziatskogo gosudarstvennogo universiteta. Novaja serija [Tachkent], 80, 12, 1956, 
Jean Chaffanjon, dont les travaux sont considérés comme l'exemple même de l'incompétence scientifique et du dilettantisme. C'est à lui que sont attribués les plus grands dommages causés à la ville ancienne pendant toute la durée de son exploration car, selon l'opinion reçue dans les milieux scientifiques, Chaffanjon étudia

« la citadelle en utilisant (pour accélérer les travaux de terrassement) la technique militaire de l'époque. À sa demande on lui détacha des sapeurs de la garnison de Samarcande qui, sur ses indications, posèrent une galerie de mines dans la couche profonde de la citadelle, y mirent de la poudre, provoquèrent une explosion et rassemblèrent ensuite les objets projetés des profondeurs de la couche culturelle vers la surface, principalement des tessons de céramique $[\ldots] . »^{14}$

Chaffanjon lui-même, le premier des chercheurs français à avoir reçu du gouvernement russe une autorisation spéciale pour mener des fouilles, décrit assez précisément dans son compte rendu la méthode choisie : «Ayant été autorisé à faire des fouilles sur l'emplacement d'Aphrosiab, j'ai procédé d'abord méthodiquement, mais les résultats furent si peu satisfaisants, que j'entrepris de faire des galeries un peu au hasard, espérant trouver un point favorable pour des fouilles réglées ${ }^{15}$ (cf. les méthodes de N. I. Veselovskij). L'utilisation de la dynamite est passée sous silence par l'archéologue français; en ce qui concerne les galeries «percées au hasard », traversant le site au petit bonheur, on peut établir une analogie - pour donner un exemple hors du site d'Afrasiab - avec la fameuse tranchée de A. V. Komarov dans la ville ancienne de Merv ou bien avec les recherches archéologiques de L. A. Zimin sur le site de Pajkend, sans même mentionner le fait que ce principe, à savoir le sondage partiel à l'aide de coupes, est utilisé avec succès encore de nos jours.

Le premier chercheur russe à faire le lien entre Samarcande et la ville grecque de Marakanda fut V. V. Krestovskij (en fait l'identification avait déjà été faite auparavant par l'érudition occidentale, elle est notamment rapportée par le voyageur américain, Eugene Schuyler ${ }^{16}$ ). Krestovskij toutefois la localisa, de façon erronée, en direction de l'ouest et du sud-ouest de la ville des Timourides « où il semblerait que l'on ait découvert des objets d'origine grecque $»^{17}$. N. I. Veselovskij, analysant les

p. 12 ; id., «K probleme istoričeskoj topografii sredneaziatskih gorodov » (Le problème de la topographie historique des villes d'Asie Centrale), Kul'tura Turkmenii v srednie veka. Trudy JUTAKE [Ashkhabad], 17, 1980, p. 102 ; V. A. Šikišin, art. cit., p. 38.

14. M.E. Masson, « K probleme... », art. cit., p. 102.

15. J. Chaffanjon, art. cit., pp. 57-58.

16. E. Schuyler, Turkistan : Notes of a journey in Russian Turkistan, Khokand, Bukhara and Kuldja, Londres, 1876.

17. V. V. Krestovskij, V gostjah u emira buharskogo (En visite chez l'émir de Boukhara), Saint-Pétersbourg, 1887, p. 41 . 
renseignements historiques concernant Samarcande et ses propres découvertes sur place, émit l'hypothèse que la Marakanda de l'époque d'Alexandre devait se trouver précisément sur l'emplacement d'Afrasiab. Il datait l'abandon d'Afrasiab de l'année 1220, date de la destruction de Samarcande par Gengis Khan. Quant à dater l'apparition d'une ville à l'emplacement d'Afrasiab, Veselovskij estime que c'est la tâche des archéologues du futur ${ }^{18}$. Chaffanjon, lui, se borne à noter que « [...] cette ville avait été détruite et rebâtie plusieurs fois ; à chaque pas on rencontre trois et même quatre modes différents de construction ou superposition. D'ailleurs, la diversité des objets qu'on y rencontre, leur style et leur origine montrent que des peuples divers et d'origines distinctes ont vécu à Aphrasiab ${ }^{19}$. Edouard Blanc est tout aussi prudent dans ses propos, il écrit que « apparemment des vestiges de la vieille ville de Marakanda mentionnée par les historiens grecs » se trouvent à Afrasiab où est situé le tombeau de Danijar ${ }^{20}$.

Pour tous les archéologues qui ont fait des fouilles à Afrasiab dans les années 1870-1890, c'était la première expérience de travail archéologique sur un site d'Asie Centrale, excepté pour Chaffanjon qui avait déjà travaillé sur le site de Pajkend. Cela explique l'absence de perfectionnement de la méthode qui risquait d'entraîner des interprétations erronées : on voulut à toute force identifier les différentes couches mises au jour (dont la plus profonde ne pouvait pas être antérieure au Moyen Age), avec toutes les périodes d'existence de la ville depuis Alexandre ${ }^{21}$; l'absence de découvertes éclatantes fit considérer comme de peu d'intérêt les recherches ultérieures sur le site; la recherche systématique des sources étrangères à l'Asie Centrale (sources grecques, sassanides, chinoises) pour les objets d'art trouvés sur le site détourna de l'analyse du contexte local de l'Asie Centrale sur lequel ils étaient apparus.

Cependant, ces premiers travaux archéologiques mirent en évidence toute la complexité de ce site aux multiples strates, ils attirèrent sur Afrasiab l'attention active des générations suivantes de savants et contraignirent à une approche plus sérieuse des fouilles archéologiques.

Les premières fouilles d'Afrasiab eurent un autre résultat significatif (et c'est bien là le reproche qu'on leur a souvent adressé), à savoir la constitution de collections archéologiques. Laissant de côté les fouilles d'amateurs, dont le produit inondait les marchés d'antiquités de la région et avait servi de point de départ aux collections privées et publiques, notons que, par les soins de V. V. Krestovskij « furent réunis 564 objets et fut constitué un album de 40 photos dont il voulait faire parvenir un exemplaire à la Commission archéologique de Pétersbourg et un autre à la

18. N. I. Veselovskij, Zapiski imperatorskogo Rossijskogo arheologičeskogo obščestva, 2, 1887, p. XCVII.

19. J. Chaffanjon, art. cit., p. 57-58.

20. E. Blanc, art. cit., p. 827.

21. Ainsi, V. V. Krestovskij avait considéré comme gréco-bactriennes des couches appartenant au Moyen Age, non antérieures au XI ${ }^{\mathrm{e}}$ siècle. 
Société archéologique de Moscou »22 mais, de ces photos, il n'a été trouvé aucune trace à ce jour. Les collections elles-mêmes furent partagées entre le musée de Tachkent et la Commission archéologique.

L'activité de collectionneur de N. I. Veselovskij lui attira de violentes attaques. Sa passion des découvertes sensationnelles lui valut le qualificatif de « chasseur de trésors ${ }^{23}$. Cependant les collections qu'il avait rassemblées à partir de ses découvertes personnelles et des acquisitions provenant d'autres collections (par exemple 1202 objets achetés chez Mirza Buhari) constituent la base des collections de l'Ermitage ou figurent dans celles des musées de Tachkent et de Samarcande.

D'après le témoignage d'Edouard Blanc, au cours de fouilles très brèves et superficielles, fut découverte une série d'objets que lui-même considère comme antiques, y distinguant en particulier des monnaies gréco-bactriennes et indoscythes ${ }^{24}$. V A. Šiškin considère, quant à lui, que ces monnaies ont plutôt été acquises auprès de la population locale ${ }^{25}$. Charles-Eugène Ujfalvy mentionne également de nombreuses découvertes de monnaies d'or, d'argent et de bronze ${ }^{26}$.

De sérieux reproches ont été également formulés à l'adresse de Jean Chaffanjon, principalement d'avoir acheté et rapporté en France la fameuse collection de L. S. Barščevskij. Selon V. A. Šiškin, la conséquence en fut que

« la Commission archéologique, ayant appris cette vente, décide de prendre des mesures particulières pour éviter la vente d'antiquités à l'étranger. C'est pourquoi en 1889 parut un ordre spécial du tsar (Sobranie uzakonenij i rasporjaženij pravitel'stva [Recueil des lois et des ordonnances du gouvernement], 43, 1889 , p. 352) ainsi qu'une circulaire du ministère des Affaires intérieures du 4 mai $1891, \mathrm{n}^{\circ} 10$. Cette circulaire, mentionnant des cas de pillage et d'exportation d'antiquités hors des frontières, recommande de permettre les fouilles 'aussi bien aux personnes privées, en particulier les savants étrangers, qu'aux membres et collaborateurs de sociétés archéologiques seulement dans le cas d'une autorisation spéciale de la Commission archéologique'. Il ne fait aucun doute que ce sont les fouilles prédatrices de Chaffanjon déjà nommé qui ont servi de prétexte à cette ordonnance. ${ }^{27}$

Mais, soyons sérieux, si Chaffanjon était à Samarcande en 1894-1895, pourquoi le décret de 1891 serait-il lié à son nom ? De quoi s'agit-il ? d'une erreur ? d'un parti pris idéologique ? ou de l'expression de la traditionnelle rivalité de l'archéologie russe à l'égard de l'archéologie occidentale?

22. V. A. Šiškin, art. cit., p. 22.

23. A. Ju. Jakubovskij, « Iz istorii arheologičeskogo izučenija Samarkanda » (Points d'histoire de l'étude archéologique de Samarcande), Trudy Otdela Vostoka Ermitaža, II, 1940, pp. 298299.

24. E. Blanc, art. cit., p. 827.

25. V. A. Šiškin, art. cit., p. 38.

26. Ch.-E. De Ujfalvy de Mezö-Kövesd, op. cit., pp. 81-82.

27. V. A. Šiškin, art. cit., pp. 44-45. 
D'autre part, Jean Chaffanjon et Gabriel Bonvalot, venus ensemble à Samarcande, disposaient d'une autorisation spéciale de la Commission archéologique impériale russe pour effectuer les fouilles d'Afrasiab aux conditions suivantes : 1. description des fouilles (avec indication sur un plan des endroits exacts des fouilles effectuées) ; 2. transmission des trouvailles antiques à la Commission archéologique impériale russe pour les présenter à l'attention du tsar ; 3 . les objets qui ne feront pas partie d'une collection de l'Ermitage impérial conformément à l'ordre du tsar seront mis à la disposition de la mission française ; 4. la Commission archéologique impériale russe garde le droit de publier les objets trouvés aux cours de fouilles ${ }^{28}$.

De toute façon, en soulevant la question de la légalité de l'exportation des collections, nous abordons un problème délicat où, à chaque opinion énoncée, on peut en opposer mille... Le problème de l'exportation des collections (aussi bien légale qu'illégale) met en œuvre un faisceau de questions juridiques, morales et philosophiques, liées à la notion de patrimoine culturel de l'humanité, aux différents paramètres de l'ouverture d'une société, au droit de la propriété privée, aux intérêts des différents États, au problème des conditions optimales de conservation du monument, aux fluctuations de la relation colonie-métropole, pays d'origine-étranger. A la fin du XIX ${ }^{\mathrm{e}}$ siècle le fait d'emporter des collections en Russie n'était pas considéré comme une exportation à l'étranger, alors qu'en cette fin de $\mathrm{XX}^{\mathrm{e}}$ siècle, pour les nouveaux pays indépendants d'Asie Centrale, la Russie apparaît comme un pays étranger aussi lointain que les pays d'Europe occidentale.

Par ailleurs, dans le travail approfondi et fondamental de V. A. Šiškin à propos des collections du chercheur français, on peut découvrir encore quelques détails qui ne sont pas sans intérêt :

«les objets recueillis par Chaffanjon à Afrasiab», affirme Šiškin, «ont été examinés par Veselovskij. D'après les conclusions de ce dernier, ce sont des fragments que l'on jette habituellement au cours des fouilles. Le seul objet est une poterie assez grossière. De cette partie de la collection qui, apparemment aurait dû rester en Russie, Veselovskij a extrait quelques monnaies d'argent des Ilek [souverains turcs médiévaux], un fragment de vase sphéro-conique recouvert de vernis bleu et 'un moule carré en albâtre dont on ignore l'usage, avec quatre petits récipients en verre encastrés à l'intérieur'. Tout le reste, Veselovskij recommande de ne pas l'envoyer à Saint-Pétersbourg mais de le laisser sur place à Samarcande pour le musée que l'on projette d'y créer. $\gg^{29}$

Ainsi, une partie des collections rassemblées par Chaffanjon devait à l'origine rester sur le territoire de l'empire russe et être mise à la disposition du musée de

28. Central'nyj gosudarstvennyj arhiv Respubliki Uzbekistan (Archives d'État de la République d'Ouzbékistan), f. I-1 (fonds de l'Administration du général-gouverneur du Turkestan), op. 11, d. 817,1.75.

29. V. A. Šiškin, art.cit., p. 40. 
Samarcande sur le point d'être créé, ou transféré au musée de Saint-Pétersbourg, ce qui modifie quelque peu l'image que l'on se fait du chercheur français. A partir de ces positions, on peut reconsidérer le jugement porté sur l'activité de Chaffanjon dans l'historiographie soviétique.

\section{Jean Chaffanjon}

On peut trouver une brève biographie de Chaffanjon dans le Dictionnaire de biographie française ${ }^{30}$. Né le 7 septembre 1854, dans la petite ville d'Arnas, dans le département du Rhône, au sein d'une famille de viticulteurs, Jean Chaffanjon acquit assez vite la réputation d'un self-made man. Après avoir combattu pendant la guerre de 1870 dans les rangs des Chemises Rouges de Garibaldi et s'être remis d'une blessure reçue dans les environs de Dijon, le jeune homme entre en 1873 à l'École Normale de Villefranche. Trois ans plus tard, en 1876, il est nommé instituteur stagiaire à Tarare, mais il refuse ce poste et obtient l'autorisation de poursuivre des études d'anthropologie à la Faculté des sciences de Lyon (1880-1882). Parallèlement, bénéficiant de la protection de son oncle Claude Bernard, membre de l'Académie et célèbre médecin français, il devint assistant naturaliste au Muséum d'histoire naturelle de Lyon. Ensuite, ce fut la Martinique, où on lui confia un poste de professeur d'histoire naturelle au lycée de Saint-Pierre - il y étudia le volcan encore en activité de la montagne Pelée -, puis l'admission à la loge maçonnique du Grand-Orient $(1884)^{31}$, la mort tragique de sa femme et de son fils, qui entraîna sa première démarche auprès du ministère de l'Instruction publique et des Beaux-Arts pour présenter un projet d'exploration pluridisciplinaire du bassin de l'Orénoque en Amérique Latine. A partir de 1884, commence pour lui une autre vie, faite de voyages et de déplacements incessants : en 1884-1890, une série d'expéditions scientifiques aux sources de l'Orénoque ${ }^{32}$, en 1894-1895, l'exploration de l'Asie Centrale et de la Sibérie, en 1898 la Sibérie orientale, la Mandchourie, la Corée ${ }^{33}$.

30. F. Marouis, « Chaffanjon (Jean) », in Dictionnaire de biographie française, Paris, Letouzey et Ané, 1933-.

31. Archives du Grand-Orient de France. Cette adhésion à la franc-maçonnerie, comme auparavant son engagement au côté de Garibaldi, montre que Jean Chaffanjon, au moins dans sa jeunesse, fut en rupture avec sa famille, très catholique et « vieille-France ». Toutefois il quittera la franc-maçonnerie en 1903.

32. Trois campagnes furent menées, la première de simple reconnaissance (1884-1885), la deuxième le long du cours supérieur de l'Orénoque jusqu'aux sources du fleuve et au lac Negro (1886-1887), la troisième, mission scientifique dans les Andes entre Colombie et Vénézuela (1888-1889 et 1890). Cf. J. Chaffanjon, Observation sur l'alligator mississipiensis, Lyon, impr. de Pitrat, 1881, 16 p. et pl. (Extrait des Annales de la Société linnéenne de Lyon, 28, 1881) ; id., «Voyage à travers les Ilanos du Caura. Voyage aux sources de l'Orénoque », in Le Tour du Monde, 56, 1889 ; id., L'Orénoque et le Caura, relation de voyages exécutés en 1886 et 1887 , Paris, Hachette, 1889, 351 p. ; id., Voyage aux sources de l'Orénoque, Paris, Société de géographie, 1888, 44 p. (Extrait du Compte rendu des séances de la Commission centrale, 14-16, 1887, Séance du 18 novembre 1887. Réception de M. J. Chaffanjon).

33. AN, fonds 17/17270/pp. 1-4. 
Comme la plupart des explorateurs de cette époque, Chaffanjon, au cours de ses nombreuses expéditions, financées en règle générale par le ministère de l'Instruction publique et des Beaux-Arts, a étudié les aspects les plus divers de ce que l'on appelait «l'histoire naturelle». Les descriptions géographiques, hydrologiques et orographiques voisinaient avec l'étude de la flore et de la faune, les descriptions ethnographiques étaient complétées par des résultats d'observations archéologiques. De plus, chaque expédition avait également pour but de rassembler des collections ethnographiques et naturalistes qui étaient ensuite transmises au Muséum d'histoire naturelle, au Musée de l'homme ou au Musée Guimet ${ }^{34}$.

Les premières expéditions de Chaffanjon à travers l'Amérique Latine (dont l'une se fit avec la participation de l'artiste Auguste Morisot) ${ }^{35}$, avaient pour but, selon l'explorateur lui-même, de « remonter le cours du fleuve, d'en réaliser une description géographique, d'étudier les mœurs et les coutumes de la population indienne vivant le long du fleuve, la faune, la flore et la géographie du bassin $»^{36}$. Ces expéditions lui apportèrent la gloire du voyageur heureux et du chercheur sérieux et lui valurent également d'être reconnu dans le milieu scientifique et dans les sphères du pouvoir ${ }^{37}$. Après les premières expéditions le long du cours inférieur de l'Orénoque, il reçoit les palmes académiques, la médaille d'or et le prix 1888 de la Société de géographie de Paris, la médaille d'or Dupleix de la Société de géographie et de commerce de Paris, ainsi que la médaille d'argent de l'Exposition universelle de 1889, sur laquelle étaient représentées les collections qu'il avait rassemblées et rapportées. Sa candidature fut proposée pour l'obtention de la Légion d'honneur. Chaffanjon refusa cette décoration pour des raisons politiques, mais accepta les distinctions du gouvernement vénézuélien - le titre de chevalier de l'Ordre du Libérateur et la médaille d'or du ministère de l'Éducation nationale ${ }^{38}$.

Son compte rendu scientifique de l'exploration de la vallée de l'Orénoque fut remarqué par Jules Verne qui suivait attentivement toutes les découvertes géogra-

34. En tout Chaffanjon transmit aux musées nationaux français en 1884 dix caisses de collections en provenance d'Amérique du sud, en 1886-1889 vingt-neuf caisses en provenance d'Amérique du sud, en 1894-1897 cinquante-trois caisses en provenance d'Asie Centrale. Parmi celles-ci figuraient des collections animales : insectes, reptiles, mammifères, des objets ethnographiques, des collections géologiques ainsi que toute une série de plantes destinées au jardin botanique du Muséum, cf. AN, fonds 17/17270/pp. 1-4.

35. J. Amaz, « Les vitraux d'Auguste Morisot », Bulletin des Musées et Monuments lyonnais, 4, 1990, pp. 22-53.

36. J. Chaffanjon, L'Orénoque et le Caura, op. cit.

37. Les résultats les plus importants de cette série d'expédition furent : la découverte des sources de l'Orénoque et la reconnaissance de la direction du cours du fleuve, la réalisation d'une série d'observations astronomiques, l'étude du système de communication hydrographique entre l'Amazone et l'Orénoque, une série d'études ethnographiques des tribus indiennes de la vallée de l'Orénoque, un ensemble de collections naturalistes et ethnographiques destinées au Muséum et au Musée de l'homme.

38. A. Chaffanjon, L'Orénoque aux deux visages. L'Orénoque et le Caura, voyage aux sources de l'Orénoque par Jean Chaffanjon. Le superbe Orénoque par Jules Verne, Paris, Denis Pierron, 1978 , p. 22. 
phiques, et servit de base à son roman Le Superbe Orénoque (1898), dont Chaffanjon est l'un des personnages. Entre l'écrivain et le voyageur s'établit une correspondance et - ironie du sort -, si Jules Verne utilisa pour son roman les notes de Chaffanjon, ce dernier, d'après la tradition familiale, étudia avec une égale attention les œuvres de Jules Verne Michel Strogoff (1876) et Claudius Bombarnac, carnet d'un reporter à l'inauguration du grand Transasiatique (1892), lors des préparatifs de son voyage en Asie Centrale.

Après un début de carrière scientifique aussi prometteur, Chaffanjon obtint sans peine l'autorisation et l'appui financier du ministère de l'Instruction publique pour organiser un voyage en Asie Centrale.

Le voyage de Chaffanjon en Asie Centrale fut financé en partie par le ministère de l'Instruction publique, en partie par son concitoyen et ami Lucien Mangini ${ }^{39}$ qui partagea plus tard sa réputation peu flatteuse dans l'historiographie soviétique. Aussi les innombrables collections d'objets d'Asie Centrale rapportés par Chaffanjon furent-elles transmises aux fonds des musées nationaux de France, alors qu'une partie de la célèbre collection de L. S. Barščevskij fut acquise par Chaffanjon aux frais de Lucien Mangini à qui elle était personnellement destinée.

\section{La collection de Barščevskij}

En 1894, L. S. Barščevskij, « séduit par l'excellente idée de la création d'un musée à Samarcande », et dans l'espoir «que les plus beaux objets ne partiraient pas à l'étranger» proposa au gouverneur militaire de l'oblast' de Samarcande, N. Ja. Rostovcev, de faire l'acquisition de sa collection « aux meilleures conditions »40 à l'intention du futur musée. Rostovcev transmit la proposition au général-gouverneur du Turkestan, le baron A. B. Vrevskij, et fit venir D. I. Evarnickij¹, à l'époque

39. Lucien Mangini (1833-1900) était une figure très en vue à Lyon. Ingénieur sorti de l'École centrale, président de la Compagnie des chemins de fer, engagé dans la construction de réseaux ferroviaires régionaux, auteur de plusieurs livres sur la construction des chemins de fer, c'était également un grand industriel, maître de forges, l'un des fondateurs et administrateurs d'une grande banque française, le Crédit lyonnais, et enfin un homme politique de premier plan : conseiller municipal en 1870, sénateur en 1876-1882, conseiller général du département du Rhône et député à l'Assemblée nationale (1871), maire de la commune de Halle. Cf. J.-P. Gutton, ed., Les Lyonnais dans l'histoire, Lyon, Privat, 1985 ; Revue du Lyonnais, 29, 5e série, 1900 ; Catalogue général des livres imprimés de la Bibliothèque nationale, 105, 1931.

40. V. A. Šiškin, art. cit., pp. 44-45.

41. D. I. Evarnickij (Javarnickij) (1855-1940), archéologue ukrainien, professeur à l'Université de Kiev, à partir de 1929 membre de l'Académie des sciences d'Ukraine, par la suite directeur du Musée d'ethnographie régionale de Dnepropetrovsk (Ekaterinoslav) auquel il fit don de sa collection archéologique. L'essentiel de ses recherches est consacré à l'histoire des Cosaques Zaporogues. Après son séjour au Turkestan, il publia un livre : Putevoditel' po Srednej Azii ot Baku do Taškenta $v$ arheologičeskom i istoričeskom otnošenijah (Guide historique et archéologique de l'Asie Centrale de Bakou à Tachkent) (Tachkent, 1983) qui s'appuie largement sur les recherches de A. Vambery, Istorija Buhary ili Transoksanii s drevnejših vremen do nastojaščego (Histoire de Boukhara ou de la Transoxiane depuis les temps les plus anciens jusqu'à nos jours), Saint-Pétersbourg, 1, 1873. 
fonctionnaire chargé des affaires spéciales à la Chancellerie du général-gouverneur du Turkestan, pour rédiger l'inventaire de la collection proposée à la vente ${ }^{42}$. La collection fut reconnue comme «l'une des plus riches collections privées de toute la région », information qui fut transmise aussi bien au général-gouverneur qu'au siège de la Société archéologique de Moscou. La collection elle-même fut évaluée au préalable à 1800 roubles.

Le baron A. B. Vrevskij, général-gouverneur du Turkestan, amateur d'antiquités et lui-même collectionneur passionné (et, un an plus tard, fondateur et président d'honneur du Cercle d'amateurs d'archéologie du Turkestan) proposa, dans une

42. G. Ja. Upfal rapporte la conclusion de D. I. Evarnickij, constituée d'une description générale et d'une énumération des objets qui composent la collection : «La collection d'antiquités du capitaine Barščevskij est constituée, à quelques rares exceptions près, d'un ensemble d'antiquités datant de la période historique avant et après notre ère et concerne en partie la période hellénistique après Alexandre le Grand, mais surtout l'époque turco-mongole, et en particulier celle de Timour. Sur ce plan la collection de Barščevskij confirme de façon évidente l'existence des deux époques découvertes auparavant et attestées dans cette région par l'orientaliste N. I. Veselovskij, professeur à l'Université de Saint-Pétersbourg. Ces objets ont été découverts principalement à Afrasiab, l'une des sources les plus importantes des antiquités du Turkestan, et dans différentes réserves archéologiques de l'est de la région de Boukhara. L'essentiel de la collection d'antiquités de Barščevskij se résume à des objets de céramique, des objets métalliques, des colliers, des objets en verre, des armes, des statuettes de dieux pénates, des médailles et des monnaies. Y figurent de plus quelques objets préhistoriques : deux couteaux de pierre, un grattoir en pierre, deux kel'ty rares par leur degré de miniaturisation, une fibule en bronze ou fermoir pour vêtement de dessus et un miroir en bronze de dimensions rares. En quantité la collection d'antiquités de Monsieur Barščevskij est l'une des plus importantes collections privées pour toute cette région. »

Dans l'énumération des objets on trouve :

«1. Jusqu'à 250 fragments d'objets de céramique ou d'argile de différentes tailles avec différents ornements et motifs caractéristiques de la région.

2. Des objets métalliques, jusqu'à 50, pots en cuivre, en bronze, en fer, plats, plateaux, brûleparfums, vases, lampes, agrafes, fermoirs, lames et autres petits objets.

3. Des colliers, 14 unités, dont deux en argent superbement travaillés et d'une longueur inhabituelle (presque deux archines chacun), le reste en pierres de toutes sortes : cornaline, améthiste, turquoise, grenat, rubis, lapis-lazuli, agathe, jaspe, ambre, ou pierre ordinaire avec des incrustations. On, doit classer ici également des anneaux en argent et en cuivre et des boucles d'oreille de différentes tailles et qualités.

4. Des récipients en verre entiers, jusqu'à 30 pièces et 300 fragments dont certains avec des inscriptions coufiques.

5. Une grande quantité de flèches en bronze, en cuivre, en fer, des boucliers, des arcs, une cotte de mailles en acier avec gantelets et genouillères.

6. Des statuettes ou idoles, jusqu'à 500, représentant des figures humaines [figurines en terre cuite-SG].

7. Des fragments de sépultures [ossuaires-SG], jusqu'à 40 unités avec des motifs d'oiseaux, de dragons, de méduses, de chameaux, d'hommes couronnés, de pleureuses.

8. Des camées, des sceaux, des amulettes, jusqu'à 80 , certains en pierres précieuses, d'autres en bronze et en cuivre.

9. Des diptyques en métal ou en os, 7 unités, représentant le Christ Sauveur, la Vierge et Nicolas le Thaumaturge.

10. Des monnaies de différentes époques et origines, jusqu'à 10000 parmi lesquelles 260 en argent, en partie hellénistiques en partie turco-mongoles. » G. Ja. Upfal, Istorija Samarkandskogo muzeja (Histoire du musée de Samarcande). Manuscrit aimablement communiqué par son propriétaire N. Almazova (Samarcande). 
lettre au président de la Commission impériale d'archéologie, comme solution optimale, l'acquisition de la collection de Barščevskij pour l'Ermitage. D'après l'information de Mme G. Ja. Upfal, conservatrice au Musée d'histoire de Samarcande, chargée d'affaire du président de la Commission, le baron V. G. Tizengauzen, dans une réponse datée du 28 juin 1894, indiquait que « le choix des objets proposés à l'acquisition ne pouvait être décidé qu'après leur examen détaillé à Saint-Pétersbourg par les membres de la Commission d'archéologie, après comparaison de ces objets avec les acquisitions précédentes auprès de collectionneurs tels Askarov ou Mirza Buhari, ainsi qu'avec les objets rassemblés par le professeur Veselovskij à Afrasiab ». Barščevskij refusa cette proposition, disant qu'il n'avait pas l'intention de vendre sa collection au détail ou objet par objet et que le transport à Saint-Pétersbourg d'objets fragiles et souvent de grande dimension, reviendrait aussi cher que la venue au Turkestan d'experts de la capitale ${ }^{43}$. D'ailleurs le Trésor ne trouva les moyens nécessaires ni pour l'une ni pour l'autre de ces solutions. En fin de compte, une partie de la collection fut achetée par le marchand moscovite, N. I. Rešetnikov, qui la remit en janvier 1896 au Comité statistique de la région de Samarcande à l'intention du futur musée de la ville ${ }^{44}$; une autre partie fut remise au même musée par Barščevskij lui-même. Le reste de la collection fut acquis pour 240 roubles par Chaffanjon ${ }^{45}$ avec qui Barščevskij avait effectué des fouilles à Afrasiab et qu'il avait accompagné à plusieurs reprises, comme il avait accompagné également un autre explorateur, Gabriel Bonvalot, à travers le Turkestan. Ainsi, si l'on tient compte de l'évaluation du coût total de la collection de Barščevskij, à savoir 1800 roubles, Chaffanjon n'a pu en acheter plus d'un cinquième. Et ce n'est en aucun cas cet achat qui a pu provoquer l'adoption par la Commission archéologique de « mesures pour éviter la vente d'antiquités à l'étranger ».

Il est intéressant de noter au passage que l'idée de vendre sa collection à la France était déjà venue à Barščevskij, qui était à la recherche de fonds pour poursuivre des travaux de prospection de gisements de charbon dans l'okrug de Samarcande. Déjà en 1885, un professeur de Grenoble, dont le nom n'a pas été retenu, s'était rendu à Samarcande à l'invitation d'une lointaine parente de Barščevskij, il avait décidé d'acheter toute la collection et avait même obtenu l'autorisation du gouvernement russe mais, pour une raison inconnue, il ne réalisa pas cet achat ${ }^{46}$. Au

43. Ibid., p. 49.

44. Dans la collection de N. I. Rešetnikov figuraient environ 1000 pièces parmi lesquelles : des objets en terracotta (434), des pendentifs (5), un sceau, des jouets en argile (17), des objets en céramique dont les plus anciens sont datés du XIVe siècle (31), des lampes des XIV-XVe siècles (3), des pots (12), des fragments d'ossuaires (56), des fragments de monuments d'architecture (30). Parmi les monnaies, on en trouve 67 en argent, 316 en cuivre et « un poud de monnaies non cataloguées ». La collection comprenait également des objets ethnographiques (G. Ja. Upfal, op. cit., p. 95). Au sujet du transfert de la collection, cf. Spravočnaja knižka Samarkandskoj oblasti na 1897 god, 5, 1897 ; Letopis' Samarkandskoj oblasti, 9 janv. 1896.

45. Journal de Jean Chaffanjon, premier cahier, p. 30, archives de la famille Chaffanjon (Paris).

46. M. M. Blombergowa, « Badania archeologiczne Polaków na terytorium Imperium Rosyjskiego w XIX i poczàtku XX wieku » (Recherches archéologiques polonaises sur le territoire de 
même moment, d'après le témoignage de Čabrov, un peu avant la vente, la collection de Barščevskij (50 pièces parmi lesquelles «des objets du l'âge du bronze », « des antiquités de l'Afrasiab » et « des objets grecs et bactriens ») ${ }^{47}$, avait été photographiée par Chaffanjon pour un album photographique qui devait être édité en France ${ }^{48}$.

Aujourd'hui il ne reste plus trace de la partie française de la collection de Barščevskij. Au Musée des Beaux-Arts de Lyon, auquel furent transmises dans les années 1960 les collections d'Asie Centrale du Musée Guimet de Lyon, est conservée l'une des plus grandes collections existant sur le sol français d'un décor architecturale provenant de monuments d'Asie Centrale. Elle est formée à partir des collections rapportées d'Asie Centrale par des explorateurs français, mais les livres d'inventaire et l'étiquetage des objets eux-mêmes ne mentionnent que les noms de Charles-Eugène Ujfalvy et Edouard Blanc. Rien n'indique la présence dans ce musée de la collection Chaffanjon-Mangini. D'autre part, les comptes rendus des séances de la Société de géographie indiquent que toutes les collections réunies par Chaffanjon furent offertes par Mangini au Muséum d'histoire naturelle ${ }^{49}$, mais là non plus je n'ai pu en retrouver aucune trace.

\section{Une mission française au Turkestan}

Mais revenons à l'expédition de Chaffanjon en Asie Centrale. Le premier projet incluait dans la composition de l'expédition l'explorateur Gabriel Bonvalot, déjà célèbre à l'époque, mais finalement leurs itinéraires se croisèrent seulement au début, notamment à Samarcande, et à l'ensemble de l'expédition participèrent seulement Chaffanjon, chef de la mission, Henri Mangini, naturaliste qui constitua une collection ethnologique et zoologique ${ }^{50}$ et Louis Gay, artiste-paysagiste et photographe, qui rassembla également une collection botanique ${ }^{51}$.

l'Empire russe à la fin du XIX et au début du XX'e siècles), Acta archaelogica Lodziensia, 37, 1993,p. 108.

47. Okraina, 14, 1895.

48. G. N. Čabrov, Častnoe kollekcionerstvo dorevoljucionnogo Turkestana (1865-1917) (Les collections privées du Turkestan avant la révolution, 1865-1917), manuscrit, Central'nyj gosudarstvennyj arhiv Respubliki Uzbekistana, f. R-2681 (archives personnelles), op. 1, d. 100,1. 10.

49. Comptes rendus des séances de la Société de géographie, Paris, 1897, p. 344.

50. Henri Mangini (1877-1901), fils unique de Lucien Mangini. Pendant ses 24 ans de vie il se fit une réputation de voyageur infatigable. Compagnon de J. Chaffanjon et G. Bonvallot, il fit plusieurs voyages au Turkestan, en Mongolie et en Sibérie et reçut les palmes académiques. Partageant l'intérêt de son père pour les chemins de fer, il avait bâti de grands projets en direction de l'Extrême-Orient russe et en particulier du transsibérien. Après le service militaire obligatoire il avait l'intention de revenir dans sa résidence de Blagoveščensk mais sa mort prématurée dans un accident de voiture empêcha la réalisation de ses projets scientifiques et commerciaux. Cf. La Revue du Lyonnais, 31, 5e série, 1901.

51. AN, fonds F 17/2946/2. 
Le but de cette mission scientifique, selon l'explorateur lui-même, était « d'explorer le Turkestan russe, les territoires du Baïkal et du fleuve Amour, d'y réunir des collections d'histoire naturelle destinées aux établissements de l'État et d'y poursuivre des recherches historiques et ethnographiques $»^{52}$.

Les premières étapes de l'expédition ne présentaient pas une grande originalité : passant par Marseille et Constantinople, les voyageurs arrivèrent à Batoum, traversèrent le Caucase et à travers la mer Caspienne atteignirent Uzun-Ata. Ils prirent ensuite la ligne ferroviaire transcaspienne, qui déterminait les itinéraires de presque tous les étrangers, explorateurs, commerçants ou simples touristes curieux. Cet itinéraire les fit passer par Achkabad, Merv, Boukhara, Karshi, Samarcande, pour arriver à Tachkent en mars 1895. Ensuite l'expédition prit la direction de Tchimkent, Aulie-Ata et du lac Issyk-Kul, atteignant la ville de Vernyj où, renonçant aux sentiers battus, ils formèrent une caravane pour la suite du voyage à travers le Sémiretchié. Ensuite le massif du Tien-Shan de Dzungarie, la Mongolie du nord et de l'est, la Mandchourie et la Sibérie orientale.

Cette expédition de près de trois ans (octobre 1894-juin 1897) dont les résultats furent importants dans les domaines de la géographie ${ }^{53}$, de l'archéologie ${ }^{54}$, de l'anthropologie ${ }^{55}$ et de l'ethnographie ${ }^{56}$, sans oublier la zoologie ${ }^{57}$, la botanique ${ }^{58}$, la géologie et la minéralogie ${ }^{59}$, fut hautement appréciée par la communauté scientifique et se vit récompensée de la médaille d'or et du prix Léon Dewez de la Société de géographie de Paris, ainsi que de deux grandes médailles Dupleix de la Société de commerce et de géographie ${ }^{60}$.

\section{J. Chaffanjon, art. cit., p. 55.}

53. Sur le tronçon de la voie ferrée Tachkent-Kuldja-Urga, ils explorèrent 400 kilomètres d'une nouvelle voie ; en Mongolie orientale, d'Urga à Blagoveščenskij, plus de 1800 kilomètres ; ils établirent des cartes orographiques des massifs montagneux et firent tout un ensemble d'observations astronomiques, indispensables au perfectionnnement des cartes géographiques existantes.

54. L'expédition réalisa des fouilles archéologiques et de reconnaissance à Merv, Pajkend, Afrasiab, où elle rassembla des collections de céramiques vernies et non vernies ainsi que des objets en bronze ; elle étudia les babas en pierre (stèles funéraires anthropomorphes) de la région d'Aulie-Ata, de Merv et du lac Issyk-Kul, dans l'Altaï, sur le bord du lac Dajn-Gol ; l'expédition réalisa également une description des ruines de Kara-Korum ; elle rassembla une collection de kajrak (pierres tombales) et de crânes dans le cimetière nestorien des environs de Pišpek ; elle étudia les constructions funéraires turques dans le nord de la Mongolie.

55. Les observations anthropologiques proprement dites furent complétées par une collecte de crânes d'origine mongole et bouriate.

56. Tout au long du voyage, l'expédition réalisa des observations ethnographiques et recueillit des collections ethnographiques (« outils agricoles, vêtements, ustensiles de ménage des habitants de Boukhara et des Sartes ; un ensemble de vêtements masculins et féminins, des bijoux de tribus mongoles et des yourtes mongoles »).

57. L'expédition rassembla des collections contenant des crânes et des os de chevaux sauvages, de chameaux, de mammifères, d'oiseaux, d'insectes, de poissons et de mollusques.

58. Un herbier fut constitué, contenant environ 2000 dénominations de plantes de la région.

59. L'expédition rapporta une collection de minéraux et de roches qui permettaient d'établir la carte géologique de la Mongolie et de la Mandchourie, des plantes fossilisées, des échantillons de charbon de bois de la région de l'Amour.

60. AN, fonds F 17/2946/2. 
Le naturaliste et voyageur Guillaume Capus, qui fit aussi le voyage d'Asie Centrale, mais seulement en tant que membre de l'expédition de G. Bonvalot (1882), rapporte que :

«Des études archéologiques dans le Turkestan puis à travers la Dzoungarie, la Mongolie et la Mandchourie, sur un parcours de près de cinq mille kilomètres, des observations géographiques qui sont parfois nouvelles, qui complètent heureusement sur d'autre points les données recueillies par d'autres voyageurs, de sérieuses observations de tout genre, voilà ce que M. Chaffanjon et ses compagnons ont exécuté pendant leur long itinéraire à travers l'Asie turcomongole. Ils ont, pour tout dire en quelques mots, su mener de front d'une manière continue l'art du cheminement et la science de la recherche [...]. L'ère des grandes découvertes étant close, une tâche plus difficile peut-être et plus délicate incombe à l'explorateur contemporain : celle de reprendre en sousœuvre l'acquit des devanciers, de combler les lacunes et de chercher dans le détail la nouveauté caractéristique et parfois déterminante que les grandes lignes n'ont pas toujours su mettre en relief. ${ }^{61}$

Chaffanjon lui-même considérait comme le résultat le plus important de son voyage les nombreuses et diverses collections qu'il en avait rapportées ${ }^{62}$. La majeure partie de ces collections, après avoir reçu la haute appréciation des professeurs du Muséum et de son directeur, E. Milne, fut présentée avec succès dans le cadre d'une exposition spéciale, aménagée dans la galerie zoologique du Muséum. L'inauguration de cette exposition en juin 1897 se fit sous la présidence du ministre de l'Instruction publique, M. Rambaud, et quelques jours plus tard elle reçut la visite des ministres du Commerce et des Colonies ${ }^{63}$.

61. A. Chaffanjon, L'Orénoque aux deux visages..., op. cit., pp. 26-27, 29.

62. De novembre 1895 à juin 1897, Chaffanjon expédia au Muséum d'histoire naturelle près de 3584 objets, le département ethnologique du Muséum reçut, en 1895, 3045 objets du Turkestan oriental ; en 1896, 5921 objets de Sibérie orientale, Mandchourie et Mongolie. Les collections rassemblées par Chaffanjon ont été étudiées par J. Martin, T. Čičerin, G. A. Poujade, P. Lesne. Ce dernier a isolé parmi les amphibiens une nouvelle variété de crabe qui, en l'honneur de l'exlorateur, a reçu le nom de « carabus chaffanjoni ». Cf. « Rapport du 8 décembre 1897 », Bulletin de la Société entomologique de France, 1897, pp. 297-300.

63. Cf. L'Événement, 30 juillet 1897 ; Petit Journal, 30 juillet 1897. Dans ce dernier on peut lire : «L'exposition de M. Chaffanjon, Henri Mangini et Louis Gay est très intéressante. L'exposition de M. Chaffanjon comprend un nombre considérable d'objets de toutes sortes, de poteries, d'ornements provenant de fouilles pratiquées à Aphrasiab, tout près du tombeau de Tamerlan ; on y voit aussi une série de costumes et de broderies, 2000 échantillons de plantes, 150 mamnifères empaillés, 800 oiseaux, une incomparable collection d'insectes, des photographies nombreuses. Notons au hasard quelques pièces particulièrement intéressantes. Ce sont quelques-unes des fameuses bombes à feu grégeois, des ornements sartes d'une grande richesse, des étoffes coloriées et des broderies d'une harmonie charmante dans le groupement des couleurs, la reconstitution exacte sur le tissu de l'épitaphe qui se trouve sur le tombeau de Tamerlan, une série de costume sartes incrustées, des objets agricoles, des costumes de Bouriates, ou Mongoles du Nord, d'autres costumes en peau de poisson [...], des fragments de statuettes et de sarcophages d'une haute conception artistique, de petits cônes de cendres humaines, [...] des moulins à prières, petits panneaux sur lesquels sont inscrits des versets du Coran et qu'il 
Malheureusement, le destin de toutes les collections rassemblées par Chaffanjon fut assez triste, de même que celui de son livre consacré au voyage dans le Turkestan. Les innombrables collections rapportées du Turkestan disparurent sans laisser de traces, du vivant même de l'explorateur64 et son livre, Souvenirs d'un voyage en Asie centrale, que Chaffanjon avait confié aux éditions Hachette, brûla dans l'incendie de la rédaction, avec les photographies, les dessins et les cartes ${ }^{65}$. Il ne reste en fin de compte, comme seuls témoignages de ce voyage, que la série de photographies remise à la Société de géographie de Paris ${ }^{66}$, un bref compte rendu rédigé d'après des notes de voyage qui ont par bonheur échappé au feu et les carnets de voyage proprement dits qui sont conservés jusqu'à nos jours dans la famille Chaffanjon ${ }^{67}$.

Les intérêts de Chaffanjon après l'expédition turco-mongole prennent un autre cours. En 1897, il rédige pour le ministère des Affaires étrangères une note sur les perspectives de développement du commerce français en Extrême-Orient où les Allemands dominaient jusque-là. Il argumente en faveur de l'ouverture d'un service consulaire français dans cette région. Il est nommé conseiller pour le commerce extérieur français à Vladivostok. En 1900, il ouvre une série de maisons de commerce à Port-Arthur, Vladivostok, Harbin. Il quitte la loge maçonnique du Grand-Orient en 1903. Il établit des relations amicales avec le commandant de la flotte russe en Extrême-Orient, l'amiral Alekseev, ainsi qu'avec le général-gouverneur de l'oblast', Duhovskij. Il vit un certain temps à Saint-Pétersbourg... Le com-

suffit de remuer quand on veut dire sa prière, $[. .$.$] , yourte ou tente mongole.... ». Chaffanjon lui-$ même dans son rapport au ministère de l'Instruction publique et des Beaux-Arts écrit que les premières pièces expédiées par lui du Turkestan contenaient des collections archéologiques provenant des fouilles d'Afrasiab et Pajkend, des fragments de céramiques et de majoliques provenant de décors architecturaux de monuments de Boukhara et Samarcande, des pierres tombales ornées de décors d'argile cuite, les estampages de Coran provenant de Samarcande, une collection d'ojets ethnographiques sartes, une collection de céramiques contemporaines et une autre de mosaïques anciennes, une yourte, des ossements de divers animaux.

64. En 1912, le docteur E. T. Hamy, membre de l'Institut et de l'Académie de médecine, professeur au Muséum et membre de la Commission des missions scientifiques, tenta de retrouver ces collections avec un intérêt tout particulier pour les pierres tombales du cimetière nestorien, mais toutes ses recherches, au Louvre, au Muséum, au Musée de l'homme, au Musée Guimet, aussi bien qu'à Bordeaux et à l'Institut restèrent vaines. Cependant, le ministère, au nom duquel Chaffanjon avait adressé toutes ses collections, en réponse à la demande du professseur Hamy, confirma que les collections avaient été transmises au Muséum et au Musée de l'homme ; l'une des caisses qui se trouvait dans les réserves du Musée de l'homme devait être transmise, sur l'initiative du directeur du musée, au Musée Guimet ou au Musée du Louvre. À l'heure actuelle, de toutes les collections rassemblées par Chaffanjon, ne sont accessibles aux chercheurs que celles qui proviennent d'Amérique du sud et qui sont conservées au Musée d'histoire naturelle. Cf. AN, fonds F 17/17270.

65. A. Chaffanjon, L'Orénoque aux deux visages..., op. cit., p. 30.

66. Ch. Deney, Le Transsibérien. Documents photographiques de la Société de géographie de Paris, Paris, Herscher, 1980.

67. Je remercie vivement Monsieur Philippe Chaffanjon, arrière-petit-fils de Jean Chaffanjon, et Madame Philippe Chaffanjon pour l'aide qu'ils m'ont apportée au cours de mes recherches. 
merce avait réussi ce que n'avait pas pu faire la politique ${ }^{68}$, prenant une part égale à celle de la science dans le registre des intérêts du chercheur français. La bonne intégration de Chaffanjon dans la société russe lui facilita la réalisation non seulement de grosses opérations commerciales, mais aussi d'une expédition scientifique en 1898 à travers la Sibérie orientale, la Mandchourie et la Corée. Elle lui permit aussi de préparer le plan détaillé d'une autre expédition pour 1907 au cours de laquelle il devait étudier, en compagnie d'Alexandre Blanc, les Indes anglaises, la région de Boukhara, l'Afghanistan et la Perse ${ }^{69}$.

La guerre russo-japonaise, qui ruina les maisons de commerce de Chaffanjon, interrompit ses activités commerciales mais ne découragea pas l'intrépide voyageur : il prit la direction de l'Indochine française où il se consacra à une chose tout à fait nouvelle pour lui, la culture de l'hévéa et la production de caoutchouc. D'après le témoignage de gens qui l'ont connu de près, il continuait à faire des projets de voyages au Turkestan et en Mongolie, mais sa mort, soudaine et absurde, ne lui laissa pas la possibilité de les réaliser ${ }^{70}$.

\section{L'héritage scientifique}

Mais revenons au maigre héritage scientifique qui s'est conservé après son premier voyage en Asie Centrale et en Sibérie orientale et, laissant de côté les recherches naturalistes et ethnographiques, arrêtons-nous seulement aux recherches historiques et archéologiques.

Le premier objectif des recherches archéologiques du chercheur français dans le Turkestan fut Merv. Il nota que, de cette antique cité détruite par l'armée de Gengis Khan, il ne restait que des fondations de murs en ruines, construits en briques crues, derrière lesquelles on devinait les traces d'une sorte d'énorme réservoir, les fondations de palais et de constructions civiles, et une mosquée construite par un souverain seldjoukide, le sultan Sanjar. Le chercheur releva également que les matériaux de la ville ancienne, de très bonne qualité, furent réemployés aux siècles suivants pour la construction d'édifices dans le village proche de Bajram-Ali. Les fouilles

68. En 1893, Chaffanjon présenta sa candidature aux élections législatives dans le département du Rhône. Cf. A. Chaffanjon, L'Orénoque aux deux visages..., op. cit., p. 24.

69. Le projet de l'expédition prévoyait le retour de Chaffanjon en France, d'où il devait rejoindre Calcutta par voie maritime et poursuivre en chemin de fer jusqu'à Peshawar, de là partir vers le nord par la route en direction de Tchitral, le long du fleuve Kop-Tep, suivre le cours de l'Amou-Daria en direction des possessions de Boukhara, puis l'Afghanistan, Kandahar, Herat et - dernière étape - la Perse en passant par Mechhed ou par l'extrémité sud de la mer Caspienne et le Turkestan russe. cf. AN, fonds F 17/17270.

70. Alors qu'il inspectait de nouvelles propriétés sur l'île de Pulan-Tjitlim dont il comptait faire l'acquisition pour les joindre à ses propres plantations, il eut la tête fracassée en tombant du bateau et mourut dans l'heure, le 5 septembre 1913. Cf. A. Chaffanjon, L'Orénoque aux deux visages..., op. cit., p. 32 . 
mirent au jour des monnaies, « de cuivre, d'argent, parfois d'or » et de nombreux fragments de céramique décorée ${ }^{71}$.

Tout aussi modestes dans l'héritage scientifique conservé de Chaffanjon sont les matériaux sur Pajkend, ville ancienne de l'oasis de Boukhara, où le chercheur français mena des travaux archéologiques pendant plus de deux mois et dont il leva un plan topographique sommaire ${ }^{72}$. Pajkend apparut aux yeux des voyageurs comme une ville morte, à la surface de laquelle « ça et là, des amas de débris de poteries mis à découvert momentanément par les vents qui charrient les sables, indiquent la grandeur et l'extension de la ville ${ }^{73}$. Tous les objets découverts sur le site - les monnaies de bronze, d'argent et d'or, la céramique ornée, les objets de bronze et de pierre - étaient, selon l'opinion de Chaffanjon qui avait montré toute la collection au président de la Société d'archéologie de Saint-Pétersbourg, le comte Bobrinskij, typologiquement proches des découvertes faites à Afrasiab.

L'expédition comporta également des aspects anthropologiques. Étudiant, en collaboration avec N. A. Pantusov et l'ingénieur Gourdet, les agglomérations et les cimetières nestoriens dans les environs de la ville de Vernyj et dans la région entre Pišpek et Tokmak (Čortorkul'), il émit l'idée (erreur typique de l'époque) que les nestoriens étaient une race inconnue et disparue ${ }^{74}$. C'est pourquoi, en plus des plans d'agglomérations nestoriennes et de pierres tombales, « ornées d'une croix grecque et d'inscriptions en syriaque », fut constituée une collection anthropologique de vingt crânes nestoriens.

Parmi les autres sites antiques superficiellement visités par Chaffanjon, on peut mentionner une ville ancienne engloutie non loin de Tur-Ajgir, à laquelle sont attachées de nombreuses légendes sur la formation du lac Issyk-Kul, « [...] on aperçoit au fond du lac, à cinq ou six mètres de profondeur, des constructions en briques cuites et, après chaque tempête, on retrouve sur les bords des fragments de poteries, des objets en fer, en bronze, etc. ${ }^{75}$. On peut signaler aussi l'ancienne capitale de Gengis Khan, Kara-Korum dans la vallée de l'Orhon dont il ne reste que « les murailles en ruines de la citadelle et une tour à moitié démolie ». D'après la description assez détaillée donnée par Chaffanjon dans son bref compte rendu, il ne s'est pas contenté d'observer superficiellement les vestiges, mais il a dû en réaliser un plan à main levée ainsi qu'un estampage des inscriptions en mongol et en chinois ${ }^{76}$. Il visita également les centres célèbres de pèlerinage musulman dans la ville

71. J. Chaffanjon, art. cit., p. 56 ; AN, fonds F 17/2946/2.

72. L'historiographie soviétique associe les premiers travaux archéologiques sur le site de Pajkend au nom de L. A. Zimin. Cf. B. V. Lunin, Iz istorii russkogo vostokovedenija i arheologii v Turkestane. Turkestanskij kružok ljubitelej arheologii (1895-1917) (Aperçu de l'histoire de l'orientalisme russe et de l'archéologie au Turkestan. Cercle des amateurs d'archéologie du Turkestan, 1895-1917), Tachkent, Fan, 1958, pp. 108-109, 126-129.

73. J. Chaffanjon, art. cit., p. 57.

74. Ibid., p. 64.

75. Ibid., p. 61.

76. Ibid., pp. 80-81. 
d'Aulie-Ata, comprenant le mausolée de Kara-Khan, le mazar (cimetière) d'AulieAta et le mazar d'Ajša-Bibi ainsi que les ruines d'Akyr-Taš non loin d'Aulie-Ata77. En ce qui concerne ce dernier monument, il faut préciser que Chaffanjon fut parmi les premiers chercheurs ${ }^{78}$ à fournir une description de ce bâtiment mystérieux qui suscite encore de nos jours des questions et des discussions parmi les archéologues et les historiens d'art. Il en a fourni le plan détaillé, qui ne s'est conservé que dans ses carnets de voyage et figurait probablement aussi dans le livre, Souvenirs d'un voyage en Asie centrale, victime des flammes.

(Traduit du russe par Yvette Lambert)

Institut des Beaux-Arts de l'Académie des Beaux-Arts d'Ouzbékistan

2, place Mustakillik

Tachkent

700029 Ouzbékistan

e-mail : gorsheni@canoe.ens.fr

77. Ibid., p. 58.

78. De brèves descriptions du monument figurent dans les travaux de P. I. Lerh, D. L. Ivanov, V. V. Bartold et V. A. Kallaur, au nom duquel on associe traditionnellement les premières étapes de l'exploration d'Akyr-Taš (1905), cf. P. I. Lerh, Arheologičeskaja poezdka v Turkestanskij kraj v 1867 godu (Expédition archéologique dans le Turkestan en 1867), Saint-Pétersbourg, 1870, pp. 28-29, 34-39 ; D. L. Ivanov, Po povodu nekotoryh turkestanskih drevnostej (À propos de quelques antiquités du Turkestan), Saint-Pétersbourg, 1885 ; V. V. Bartol'd, Otčet o poezdke v Srednjuju Aziju s naučnoj cel'ju v 1893-1894 gg. (Compte rendu d'un voyage à visée scientifique en Asie Centrale en 1893-1894), Saint-Pétersbourg, 1897 ; V. A. Kallaur, «Poezdka na Akyr-Taš i ego okrestnosti » (Voyage à Akyr-Taš et ses environs), Protokoly Turkestanskogo kružka ljubitelej arheologii, 10, 1905, pp. 29-30, 37 ; S. Hmel'nickij, Meždu arabami i tjurkami (Entre Arabes et Turcs), Berlin - Riga, 1992,pp. 214-216 ; K. M. Bajpakov, A. Northedž, « Novye dannye ob Akyr-Taše » (Nouvelles données sur Akyr-Taš), Izvestija Ministerstva nauki i Akademii nauk Respubliki Kazahstana, Serija obščestvennye nauki, 1, 1997,pp. 83-93. 